\title{
La investigación como estrategia pedagógica para el reciclaje en la IED Rural Guillermo Alvarez ${ }^{1}$
}

\section{Research as a pedagogical strategy for recycling in the Rural IED Guillermo Alvarez}

DOI: http://dx.doi.org/10.17981/cultedusoc.9.3.2018.60

Artículo de investigación. Fecha de recepción: 15/06/2018. Fecha de aceptación: 27/11/2018

\author{
Jorge Hurtado-Ibáñez; \\ Ángela Castro-Vega, Carlos Escalante-Hernández, \\ Silvia Caballero-Noriega; Wilson Perea-Ospino; \\ Elizabeth Pisciotti-Orozco; José Peñaranda-López; Juan Alzamora-Isaza; \\ Mary Herrera-Loaiza; María Rivas-Tamayo y Tedis Badillo-López ${ }^{3}$ \\ Institución Educativa Departamental Rural Guillermo Álvarez (Colombia) \\ profe-jorge2008@hotmail.com
}

Para citar este artículo:

Hurtado-Ibáñez, J., Castro-Vega, A. Escalante-Hernández, C., Caballero-Noriega, S., Perea-Ospino, W., Pisciotti-Orozco, E., PeñarandaLópez, J., Alzamora-Isaza, J., Herrera-Loaiza, M., Rivas-Tamayo, M. y Badillo-López, T. (2018). La investigación como estrategia pedagógica para el reciclaje en la IED Rural Guillermo Álvarez. Cultura. Educación y Sociedad 9(3), 505-512. DOI: http://dx.doi.org/10.17981/ cultedusoc.9.3.2018.60

\section{Resumen}

La investigación para muchas personas se considera algo aburrido y tedioso, por la complejidad de los temas estudiados. Pero es un proceso que ayuda a desarrollar las destrezas investigativas tanto en el ámbito ecológico como ambiental, al buscar alternativas con un pensamiento reflexivo, crítico y exploratorio sobre los fenómenos ambientales que pasan en su cotidianidad. A nivel mundial, tanto en Latinoamérica como el Caribe, el manejo de la basura y su posterior utilización son un problema que afecta a todos. El presente artículo plantea como la investigación se puede utilizar como una estrategia pedagógica (IEP) para el reciclaje. Se centró bajo el enfoque cualitativo, desde el tipo de investigación acción. La población de este estudio estuvo constituida por cincuenta (50) estudiantes del grado sexto de bachillerato de la IED Rural Guillermo Âlvarez. Dando como resultados la realización de actividades pedagógicas con las cuales se identificaron las concepciones y hábitos de los estudiantes con respecto al reciclaje dentro de la institución. Llegando a las respectivas conclusiones de la necesidad de Reciclar, Reutilizar y Reducir las basuras generadas por la institución.

Palabras clave: Educación, investigación, reciclaje, medio ambiente, basura,

\section{Abstract}

Research for many people is considered boring and tedious, due to the complexity of the topics studied. But it is a process that helps to develop research skills both in the environmental and environmental field, when looking for alternatives with reflective, critical and exploratory thinking about the environmental phenomena that happen in their daily lives. At the global level, both in Latin America and the Caribbean, the handling of garbage and its subsequent use are a problem that affects everyone. This article presents how research can be used as a pedagogical strategy (IEP) for recycling. It was focused under the qualitative approach, from the type of action research. The population of this study was constituted by fifty (50) sixth grade students of the IED Rural Guillermo Álvarez Baccalaureate. Giving as results the accomplishment of pedagogical activities with which the conceptions and habits of the students with respect to the recycling inside the institution were identified. Arriving at the respective conclusions of the need to Recycle, Reuse and Reduce the garbage generated by the institution.

Keywords: Education, research, recycling, environment, garbage.

1 Este articulo ha sido derivado del programa de fortalecimiento de la cultura ciudadana y democrática CT+I a través de la IEP apoya en TIC en el Departamento de Magdalena: CICLON

2 Líder del grupo de investigación "Los Investigadores".

3 Docentes de la Institución Educativa Departamental Rural Guillermo Álvarez y miembros del Grupo de investigación "Los Investigadores”.

- The author; licensee Universidad de la Costa - CUC.

Cultura, Educación y Sociedad vol. 9 no. 3, pp. 569-578. Diciembre, 2018

Barranquilla. ISSN 2389-7724 Online 


\section{Introducción}

La única forma contundente de acercarse a unas respuestas certeras o, al menos, aceptables es la investigación, la cual nos conduce al camino de la reflexión, sobre determinados temas, específicos y amplios, despertando nuestra curiosidad y hasta el asombro sobre ellos. Al desconocer un tema, es necesario investigar y en la vida cotidiana no prestamos atención a este adagio y nos apresuramos a sacar apresuradas conclusiones. En la vida escolar no debe suceder este acontecimiento; el estudiante debe utilizar la investigación para satisfacer con resultados verificables o al menos con información verificando su apetito intelectual.

En América Latina, Colombia se puede considerar uno de los países donde la educación hace poco énfasis en enseñar a sus niños la importancia que tiene la investigación dentro de sus procesos educativos y formativos. Con una generación que emplean en la mayoría de los casos el internet para dar respuesta a las tareas investigativas asignadas por sus docentes, sin refutar ninguna de la información adquirida a través de este canal de transmisión de conocimiento; solo empleando acciones como las de copiar y pegar dentro de sus trabajos escolares.

La investigación a estos niveles debe ser concebida similar al pensamiento estratégico que según Arellano (2004) citado por Mendoza \& López (2015), "hace referencia a la forma del pensamiento que se dirige hacia la creatividad, imaginación, y búsqueda de opciones innovadoras y realistas, como potenciación de lo posible enmarcado dentro de un proyecto de construcción intencional de la realidad". (p. 154), con lo cual como lo expresan Maculan, Jiménez y Castellanos (2015) se podrá avanzar en el conocimiento.

La Institución Educativa Departamental (IED) rural Guillermo Álvarez y en general el departamento del Magdalena (Colombia) no son ajenos a esta problemática demostrando como sus instituciones dan poca importancia a la investigación como un proceso estratégico, pedagógico y educativo, con una desmotivación generalizada dentro del estudiantado de la región hacia estas actividades formativas, por su desconocimiento sobre este tema, baja comprensión lectora y poca información docente dentro y fuera de las aulas de clase.

En la actualidad existe cierta diversidad en propuestas enfocadas en estas metodologías pedagógicas, en las cuales se plantea a la investigación como fuente del conocimiento dentro de la variedad de temas que contemplan desde la sicología como el medio ambiente dependiendo de las corrientes donde se aplican. Esta teoría basada en investigación se encuentra implícitas en la Enseñanza por descubrimiento y el Programa Ondas, con su propuesta de la Investigación como Estrategia Pedagógica (IEP), con una perspectiva que se desarrolla dentro de ese enfoque y emplea elementos de la pedagogía critico-liberadora y socio-cultural (Mejía y Manjarrés, 2010). Es importante comprender la estrategia como un dinamismo que potencializa el crecimiento socioeconómico, que genera desarrollo e inclusión (Pacheco, 2013).

Esta metodología tiene sus inicios en los años ochenta (80) en EE.UU., con Jerome Bruner como promotor, y llegó a España, donde algunos profesores de la Universidad de Valencia y Barcelona (Porlán, 1995), iniciaron su aplicación en las escuelas de sus países. Reconocimiento la forma de tratar ciertos problemas donde los niños y científicos tienen una organización con procesos complementarios y semejantes. A través de ellos es susceptible su aplicación en el trabajo de los grupos infantiles, para lograr con ellos, por sus propios medios y mediante la re- 
flexión, se pueda descubrir la estructura de la realidad, como teorías, procesos, modelos y productos de la ciencia

Los contenidos de todos estos procesos educativos se vuelven problemas por solucionar, y a partir de las diferentes preguntas que se generan se estructura el currículo, en donde los avances de los estudiantes se determinan por la ampliación de estructura que se manifiesta a través del lenguaje, el raciocinio y el conocimiento. Dicho descubrimiento no es autónomo, sino que se hace bajo la guía del profesorado encargado, quienes se encargan de planificar los ejercicios y los procedimientos para alcanzar el fin buscado, el conocimiento (Pozo, 2006).

Este proceso de aprendizaje se fundamenta en 8 principios (Ramírez-Molina, 2018):

a. La capacidad que existe para resolver problemas como la meta principal de la educación.

b. El entrenamiento en la heurística es más importante que la entrega de contenidos sin significado.

c. El descubrimiento es el camino para enseñar los contenidos curriculares en las instituciones de educación.

d. Cuando el aprendizaje se hace por descubrimiento, organiza de manera eficaz lo aprendido.

e. El conocimiento verbal es la clave de cualquier transferencia tanto de información como del mismo conocimiento.

f. El descubrimiento por si es el fundamento de la autoconfianza y la fuente de motivación.

g. El descubrimiento asegura la conservación del recuerdo.

h. El descubrimiento construye a cada joven como pensador crítico. (p. 4)

Teniendo como fundamento estos 8 principios, el método debe reconocer siempre que el proceso cognitivo tiene 3 etapas (Ramírez-Molina, 2018): a. Enactivo: es la primera inteligencia práctica. Surge del contacto con los objetos y con los problemas de acción.

b. Icónico: es la representación a través de imágenes. Es libre de acción.

c. Simbólico: combina los dos anteriores y se convierten en un lenguaje (p. 5).

Aplicando estas dinámicas tanto el maestro como el estudiante que se retroalimentan con estos ejercicios producen cambios en su práctica pedagógica con la búsqueda de la certeza como principio y fin de la investigación. Creando no científicos sino personas más justas y menos desiguales con una formación en valores que amplifican las capacidades humanas (cognitivas, afectivas, valorativas y de acción) sobre las cuales está fundamentada la investigación como estrategia pedagógica.

El segundo hecho es el lugar que el trabajo intelectual ha tomado en esta sociedad, al ser convertido en un generador de valor y como un elemento central para construir el capitalismo, así como también lo que se conoce como protección de los individuos en torno a las competencias de los mercados, enmarcado en las necesidades humanas satisfechas e insatisfechas a la que nos enfrentamos en estos tiempos de cambio, transformando a los que trabajan con el conocimiento en sujetos claves de los nuevos procesos, alcanzando a todos los educadores, de todos los tipos de conocimiento (Mejía, 2011 y Herrera, 2013).

Es un ejercicio que requiere que todo educador se reconozca como actor en estas nuevas condiciones, lo cual exige un replanteamiento de las anteriores formas de educación y modifica su nueva condición. En efecto, su subjetividad y los escenarios de su acción se vuelven campos remasterizados y, por lo tanto, en disputa para construir un proyecto de emancipación o de simple modernización al servicio 
de los grupos que dominan en esta sociedad. Es allí donde el educador se pone en escena con sus capacidades humanas al servicio de los intereses precisos y concretos de las instituciones educativas, más exactamente de sus estudiantes (Cetrulo, 2001).

La tercera premisa que falta por reconocer es cómo la investigación se convierte en uno de los aspectos centrales en la configuración de un trabajo inmaterial, dando forma a este nuevo proyecto de control del capital, siendo uno de los apartados que da más valor a este proceso educativo. Ahí se da a reconocer el lugar de esta, la investigación como factor clave en la configuración de la revolución del conocimiento en la cual se encuentra. A la vez que genera condiciones nuevas en la presente sociedad, y se constituye a sí misma como campo del entendimiento, generando saberes y aproximaciones diversas desde y hacia ella.

Al percibirlo de esta forma, la investigación desde el punto de vista de la crítica educativa enmarca una lucha entre la teoría y la práctica por la forma en como el conocimiento enmarcan una acción que durante años había sido señalado como objetiva, y que ha construido una forma de ella, y ha ayudado a generar formas de poder que en la sociedad han servido para el control y la gestación de las desigualdades existen hoy en día (Ortega, 2009).

Desde la perspectiva del medio ambiente, esta lucha ha desencadenado una serie de dificultades a nivel global, por la necesidad imperante de emplear este conocimiento que se está adquiriendo para el cuidado de la naturaleza. La cual se ha visto afectada por la falta de interés de las formas de gobierno y de la población mundial por aplicar el conocimiento en beneficio de la naturaleza y del planeta en el cual se habita en la actualidad. Para Medina (1999):
El medio ambiente es un tema estudiado por muchos ya que afecta tanto a niños, jóvenes, adultos sin importar la raza, el género o la nacionalidad; la contaminación es cada vez mayor y poco o nada hacen los seres humanos para disminuir esta gran problemática ( $p$. 13).

Actualmente se está viviendo en una sociedad que presenta una transformación radical a nivel de conocimiento, cultura, sociedad, tecnología y medio ambiente para la construcción de unas mejores condiciones de convivencia y calidad de vida para los ciudadanos. Así, la cultura ciudadana "se expresa a través de las formas de organización y de convivencia social y de la manera de transformar los recursos naturales para la satisfacción de las necesidades e intereses humanos" (Alaniz, 2016, p. 10)

El proceso de reciclaje de los productos de acuerdo con las normas vigentes no es de carácter obligatorio, únicamente para aquellos municipios o ciudades que tengan más de 8.000 usuarios están obligados a realizar análisis de viabilidad de proyectos de aprovechamiento, y en aquellos casos en que dichos análisis demuestren ser sostenibles económica y financieramente, es el municipio quien estará en la obligación de promoverlos (Decreto 1713 de 2002).

Con estas leyes se puede decir que las ciudades de hoy en día se constituyen como el paradigma de la imprevisión y de la especulación, es decir, de la insostenibilidad a nivel ecológico. Haciendo necesario que generar este proceso de cambio en la cultura ambiental de la comunidad según dicen Vilches y Gil (2007), ya que la educación debe ayudar en este proceso crucial de transformación, para lograr formar para la sostenibilidad. Para Girardit (2001): 
A 7 finales del siglo XX la humanidad se halla inmersa en un experimento sin precedentes; nos estamos convirtiendo en una especie urbana. Las grandes urbes, no los pueblos ni las pequeñas ciudades, se están convirtiendo en nuestro hábitat principal. Será en las ciudades del siglo XXI donde se decida el destino humano y donde se dicte el destino de la biosfera. No existirá un mundo sostenible sin ciudades sostenibles. ¿Podemos construir un mundo de ciudades medioambiental, social y económicamente viables a largo plazo? (Girardet, 2001)

\section{Metodología}

\section{Diseño}

La investigación en la Institución Educativa Departamental - IED rural Guillermo Álvarez cuenta con una metodología flexible en la cual se escogerán una muestra representativa de los estudiantes de la Básica secundaria de la jornada de la tarde y después de realizar varias actividades se aplicaran instrumentos de recolección de información y así determinar si cuando mejora el ambiente escolar también mejoran los procesos como la enseñanza y el aprendizaje de la educación ambiental; se espera además que los estudiantes se concienticen sobre la importancia de reciclar los residuos sólidos y convertirlos en elementos para mejorar el ambiente en su escuela de forma creativa.

\section{Participantes}

Para tal efecto se escogieron muestras de treinta (50) estudiantes de 6 grado de básica secundaria de la Institución Educativa Departamental - IED rural Guillermo Álvarez ubicados en la Zona Bananera del departamento de Magdalena. Los estudiantes cumplieron con el diligenciamiento del consentimiento informado.

\section{Técnicas e Instrumentos}

La selección y elaboración de los instrumentos para la recolección de información es pieza fundamental para el proceso de la recolección de datos, ya que la falta de ellos resulta en una tarea imposible de realizar al no tener acceso a la información que se necesita para poder realizar la sistematización de los datos obtenidos. Se empleó la modalidad de observación participante donde el fenómeno se conoce desde adentro, dándole sentido al docente, quien es el que conoce y comparte con los estudiantes a diario. Por medio de este procedimiento se pueden conocer todos los aspectos y definiciones que posee cada individuo sobre la realidad que organiza sus mundos.

\section{Resultados}

El eje de esta propuesta de investigación parte de la idea de mejor el ambiente ecológico de la institución educativa fomentando en los estudiantes estrategias pedagógicas de investigación, los cuales se encuentran en permanentemente negociación con las formas establecidas del conocimiento; fomentando la investigación en los grupos infantiles y juveniles que rodean a la población participante con la búsqueda de la unidad en la relación de los saberes y conocimientos en donde ayude a complementar y que siga creciendo esta estrategia dentro de la institución, a través de una propuesta metodológica que refuerza el reconocimiento social de los actores, quienes son los que ponen en juego esos diferentes conceptos, que podrán tramitar reconociendo la visibilidad de múltiples métodos investigativos, en coherencia con el tipo de problema.

De esta investigación y partiendo de las anteriores premisas se obtienen los siguientes resultados (tabla 1 ). 
TABLA 1

Sintesis de los resultados alcanzados

Resultados

Concientización de los estudiantes frente al cuidado del medio ambiente.

Decoración de la institución con mensajes alusivos al reciclaje.

Creación de un centro de acopio para los desechos en la escuela.

Clasificación de los desechos recolectados.

Creación de elementos decorativos a partir de los productos reciclados.

Optimización del ambiente escolar por parte de docentes y estudiantes.

Fuente: elaboración propia, 2018.

Se realizaron jornadas de aseo dentro y en los alrededores de la institución, con la participación de los distintos grupos de estudiantes bajo el acompañamiento de los padres y docentes. Actualmente, se encuentra en el proceso de reciclar todos los residuos sólidos, como el plástico de los envases de agua y bebidas azucaradas, los cuales serán reutilizados en la elaboración de materas y canecas para el depósito de los deshechos generados en la institución. En ese mismo sentido, uno de los pilares fundamentales para la ejecución de este proyecto, ha sido el trabajo que los profesores de las diferentes áreas, realizado durante las clases, donde se logró que los estudiantes se apropiaran del espacio institucional en el cuidado y conservación del ambiente, dentro y fuera del aula de clase.

\section{Discusión}

De esta investigación son muchas las expectativas en cuanto a la aplicación y ejecución del proyecto, las cuales fueron alcanzadas con amplios beneficios para la institución y la comunidad en gene- ral del departamento del Magdalena. Empezando por el grado de pertenencia existente en el estudiantado hacia la institución educativa en materia de reutilización de los recursos reciclables.

Los estudiantes se apropiaron de conceptos como el reciclaje, el medio ambiente, los deshechos y el clima escolar; provocando una optimización del ambiente escolar, lo cual se vio reflejado en mejoras en los procesos educativosecológicos que se llevan a cabo en la escuela.

Provocando que la sede educativa fuera embellecida con el Mantenimiento de toda la institución por parte de docentes y estudiantes, aprovechando las materias primas que tenían a su alcance, al ser reutilizadas como ornamentación de la misma. A partir de los anteriores aspectos se puede decir que las malas prácticas ambientales que se venían llevando en la Institución Educativa Departamental - IED rural Guillermo Álvarez mejoraron sustancialmente con la realización de la presente propuesta.

Al realizar actividades entre los docentes, los padres de familia y los respectivos estudiantes de la institución se hizo una integración de estos con los miembros de la comunidad educativa del plantel y la ciudadanía en general involucrando a estos en la solución de los problemas que se presentaban en los aspectos ambientales, ecológicos y sociales, dando unas soluciones adecuadas de una forma grata y satisfactoria. Por todos estos aspectos se puede decir que el reciclaje es la mejor manera de sacarle provecho a los materiales utilizados para crear otros nuevos productos, ya que con esta práctica se puede contribuir a que todo nuestro alrededor esté más limpio y de esta manera tener un clima saludable para el aprendizaje de los educandos. 


\section{Referencias}

Alaniz, E. (Septiembre 14, 2016). La cultura ciudadana, protectora del medio ambiente. Agrofy news. [Interes general]. Recuperado de http://news.agrofy.com.ar/noticia/160791/ cultura-ciudadana-protectora-medioambiente

Cetrulo, R. (2001). Alternativas para una acción transformadora: Educación popular, ciencias y política. Montevideo: Trilce-Instituto del Hombre.

Girardet, H. (2001). Creando ciudades sostenibles. Valencia: Tilde.

Herrera-Tapias, B. (2013). La constitucionalizarían de los derechos del consumidor en Colombia: un análisis desde los derechos sociales fundamentales. Revista Civilizar Ciencias Sociales Y Humanas. 13(25). 33-48. Recuperado de http://www.scielo.org. co/pdf/ccso/v13n25/v13n25a04.pdf

Maculan, A.-M., Jiménez , C. y Castellanos, O. (2015). Aprendizaje en el proceso de incubación de empresas de base tecnológica. Económicas CUC, 36(1), 15-48.http://dx.doi.org/10.17981/ econcuc.36.1.2015.21

Medina, M. (1999). Reciclaje de desechos sólidos en América Latina. Frontera Norte, 11(21). 7-31. http://dx.doi. org/10.17428/rfn.v11i21.1411

Mejía, M. R. (2011). Las escuelas de la globalización. El conflicto por su reconfiguración. Bogotá, D.C.: Desde Abajo.

Mejía, M. R. y Manjarrés M. H. (2010). Las pedagogías fundadas en la investigación. Búsquedas en la reconfiguración de la educación. +Revista Internacional Magisterio, 42(1). 16-26. Recuperado de http://eduteka.icesi. edu.co/pdfdir/PedagogiasInvestigacion.pdf
Mendoza, D. y López, D. (2015). Pensamiento estratégico: centro neurálgico de la planificación estratégica que transforma la visión en acción. Económicas CUC, 36(1), 153-179. http:// dx.doi.org/10.17981/econcuc.36. 1.2015 .26

Ortega, P. (2009). Sujetos y prácticas de la pedagogía crítica. Bogotá, D.C.: El Búho.

Pacheco, C. (2013). Estrategias empresariales más utilizadas por las pymes en Sincelejo. Económicas CUC, 34(1), 183-202. Recuperado de https://revistascientificas.cuc.edu.co/economicascuc/article/view/583/pdf_75

Porlán, R. (1995). Constructivismo y es cuela: hacia un modelo de enseñanza aprendizaje basado en la investigación. Sevilla: Diada.

Pozo, J. y. (2006). Aprender y enseñar ciencia. Madrid: Morata.

Ramírez-Molina, R. (2018). La Investigación como Estrategia Pedagogica en las Instituciones Educativas de Macondo. Barranquilla: Ciclón.

Ramírez-Molina, R. (2018b). El manejo de los Recursos sólidos para la creación de ambientes óptimos de aprendizaje en las Instituciones de Educación Departamental. Barranquilla: Ciclón.

República de Colombia. Ministerio de Educación. (6 de agosto de 2002). Por el cual se reglamenta la Ley 142 de 1994, la Ley 632 de 2000 y la Ley 689 de 2001, en relación con la prestación del servicio público de aseo, y el Decreto Ley 2811 de 1974 y la Ley 99 de 1993 en relación con la Gestión Integral de Residuos Sólidos. [Decreto 1713]. Diario Oficial: 44893. Recuperado de http://www.alcaldiabogota.gov.co/ sisjur/normas/Norma1.jsp?i=5542 
Vilches, A. y Gil, D. (2007). Emergencia planetaria: Necesidad de un planteamiento global. Educatio Siglo XXI, 25

(1). 19-49. Recuperado de https://www. uv.es/Vilches/documentos\%20enlazados/Emergencia\%20planetaria\%20 Educatio.pdf 